\title{
Nuevos Territorios de PAZ. APORTES Y RECOMENDACIONES PARA LA TERRITORIALIZACIÓN DE LOS ACUERDOS Y LA IMPLEMENTACIÓN DE LA REFORMA RURAL INTEGRAL
}

\author{
Carlos Madridejos Ornilla* \\ Yolanda Salinas CoY**
}

\section{Resumen}

El programa Nuevos Territorios de Paz, implementado durante los últimos seis años por la Unión Europea y el Gobierno nacional, ha impulsado iniciativas de construcción de paz en cuatro regiones del país fuertemente afectadas por el conflicto armado. Bajo un enfoque territorial y de intervención multinivel, ha promovido nuevos modelos de diálogo y articulación con el objetivo de generar condiciones para la gobernanza democrática, la reducción de conflictividades sobre el acceso y uso de la tierra, y la promoción de modelos productivos sostenibles bajo criterios de preservación medioambiental e inclusión socioeconómica. Ante la implementación de los acuerdos de paz es necesario visibilizar y capitalizar los aprendizajes acumulados durante el programa, los cuales representan insumos valiosos para la territorialización de las políticas públicas y la implementación de la reforma rural integral. El presente artículo no trata de visibilizar los resultados del programa o las conclusiones de su evaluación, sino que intenta transmitir, desde una mirada crítica y basada en el ejercicio práctico, los aprendizajes más relevantes para la estructuración de nuevas intervenciones, estrategias y programas en el marco del posconflicto.

\footnotetext{
* Magíster en Acción Solidaria Internacional de Europa. Asesor en Gestión del Conocimiento. Equipo de Asistencia Técnica de la Unión Europea para el programa Nuevos Territorios de Paz. [carlos.madridejos@gmail.com].

** Especialista en Gestión del Desarrollo Regional. Asistente técnica. Equipo de Asistencia Técnica de la Unión Europea para el programa Nuevos Territorios de Paz. [workyoli@gmail.com].

Recibido: 29 de agosto de 2017/ Modificado: 21 de febrero de 2018/ Aceptado: 22 de febrero de 2018.

Para citar este artículo

Madridejos Ornilla, C. y Salinas Coy, Y. (2018). Nuevos Territorios de Paz. Aportes y recomendaciones para la territorialización de los acuerdos y la implementación de la reforma rural integral. OPERA, 22, pp. 5-27.

DoI: https://doi.org/10.18601/16578651.n22.02
} 
Palabras clave: posconflicto, Unión Europea, desarrollo local, desarrollo territorial, inclusión socioeconómica, competitividad territorial.

\section{NEW TERRITORIES OF PEACE. CONTRIBUTIONS AND RECOMMENDATIONS FOR THE TERRITORIALIZATION OF THE PEACE AGREEMENTS AND THE IMPLEMENTATION OF THE INTEGRAL RURAL REFORM}

\section{Abstract}

The New Territories of Peace program, implemented over the last six years by the European Union and the National Government of Colombia, has promoted peacebuilding initiatives in four regions of the country heavily affected by the armed conflict. Under a territorial and multi-level intervention approach, the program encouraged new models of dialogue and articulation with the aim of generating conditions for democratic governance, reducing conflicts over access and use of land while promoting sustainable productive models based on criteria of environmental preservation and socioeconomic inclusion. In the framework of farc Peace Agreements, we deem necessary to make visible and capitalize on the accumulated learning process of this program. It stands as a valuable input for the territorialisation of public policy and the implementation of the Comprehensive Rural Reform. This article does not seek to present the programs' results or its evaluation. It rather attempts to convey, from a critical perspective and based on the practical exercise, those appearing as the most relevant lessons for the structuring of new interventions, strategies and programs in postconflict.

Key words: Postconflict, European Union, local development, territorial development, socioeconomic inclusion, territorial competitiveness.

Desde hace más de veinte años la Unión Europea (UE), a través de sus instrumentos de cooperación internacional y acción humanitaria, ha venido impulsando iniciativas de construcción de paz en diferentes regiones de Colombia y ha destinado recursos financieros y técnicos para los procesos de fortalecimiento institucional, apoyo a los movimientos sociales, desarrollo rural y promoción del diálogo Estado-región. El programa Nuevos Territorios de Paz (en adelante, NTP), implementado desde el año 2011 por la UE y Gobierno nacional, ha permitido recoger los aprendizajes acumulados durante dos décadas y poner en marcha un modelo de intervención multinivel en los departamentos de Caquetá y Guaviare, y en las subregiones de Canal del Dique y Bajo Magdalena. La inversión, que ha superado los 38 millones de euros, ha contado con la participación de agencias de Naciones Unidas, organizaciones de la sociedad civil, entidades públicas y actores locales, y representa un ejemplo muy valioso para la territorialización de los acuerdos de paz, la dinamización de las economías locales y la reducción de las conflictividades. Es necesario tener en cuenta que el programa inicia sus actividades en medio del enfrentamiento armado (2011), desarrolla su intervención durante las negociaciones de La Habana (2012-2016), y concluye acciones 
en el marco del posconflicto (2016-2017), lo que le ha otorgado una visión integral e intersectorial de las oportunidades, amenazas y retos que enfrenta la consolidación de paz en el ámbito local y comunitario. Colombia enfrenta un momento histórico y durante los próximos meses avanzará, bajo el liderazgo de la nueva institucionalidad, en ambiciosos ejercicios de planificación, inversión e intervención que permitan revertir la exclusión histórica de las regiones más afectadas por la guerra y asentar las bases de la denominada "paz territorial". Este concepto, adoptado por la Oficina del Alto Comisionado para la Paz, ha ocupado un lugar protagónico en el proceso de negociación y ha situado en el centro del debate la necesidad de aplicar un enfoque diferencial en la implementación de los acuerdos, con el objetivo de adaptarlos a la heterogeneidad de los territorios, a sus potencialidades $\mathrm{y}$ a las diferentes expresiones de la violencia que el conflicto y las economías ilícitas han generado en las comunidades. El proceso de posconflicto está estrechamente ligado a una movilización de los actores locales, al establecimiento de un nuevo diálogo entre centralidad y regiones periféricas, y a la búsqueda de visiones compartidas o consensos locales como eje de articulación. Colombia, a pesar de su diversidad y heterogeneidad, se ha administrado tradicionalmente desde la capital, con un enfoque predominantemente centralista, $y$ ha otorgado a los gobiernos subnacionales, las entidades territoriales y las comunidades locales un rol pasivo y secundario en el diseño de las políticas públicas y la priorización de las inversiones. Este esquema de trabajo, junto con los problemas estructurales de acceso, concentra- ción y uso de la tierra, ha generado relaciones antagónicas entre el Gobierno nacional y los territorios, lo que se expresa también en modelos económicos en continuo conflicto con las comunidades locales. Teniendo en cuenta que una de las raíces del conflicto armado es el elevado índice de pobreza y desigualdad en los entornos rurales, abordado en el punto 1 de los acuerdos de paz, parece necesario avanzar hacia nuevos modelos de descentralización, que no solo deleguen competencias a los territorios sino que sean acompañadas de recursos y de capacidad para la toma real de decisiones en términos de inversión y desarrollo económico. Tal y como menciona la Redprodepaz en una de las publicaciones del programa:

La participación ciudadana debe comprenderse como un elemento fundamental y su promoción como una tarea sustantiva de la gestión pública en todos los niveles territoriales. Las prácticas participativas contribuyen a la construcción de soluciones a los problemas que afectan a la ciudadanía, atendiendo las especificidades territoriales, y las características y capacidades de cada región y de los diversos grupos sociales que la integran (Maldonado, 2016).

Bajo estos parámetros, es muy significativo capitalizar los aprendizajes, positivos y negativos, del programa Nuevos Territorios de Paz y trasmitirlos a la nueva institucionalidad y a los actores de desarrollo involucrados, los cuales pondrán en marcha los procesos de planeación participativa, movilización ciudadana y gobernanza local. Entre estos instrumentos es necesario destacar los planes de desarrollo con enfoque territorial (PDET) que articularán las inversiones en los 170 municipios prioriza- 
dos por el Gobierno nacional y que requieren elaborar de manera participativa un plan de acción para la transformación regional, que incluya todos los niveles del ordenamiento territorial, que sea concertado con autoridades locales y comunidades, y que contemple tanto el enfoque territorial de las comunidades como un diagnóstico objetivo de necesidades y acciones en el territorio, así como metas claras y precisas para su propósito (Ministerio de Agricultura y Desarrollo Rural, 2017). Esta herramienta servirá de base para la implementación de la reforma rural integral (RRI), punto 1 de los acuerdos, y se diseñará a través de ejercicios de participación multinivel, desde el nivel veredal hasta el subregional, que permitan integrar los consensos locales en Planes de Acción para la Transformación Regional (PATR). Más allá de una evaluación final, el presente artículo pretende abordar algunas de las principales conclusiones del programa Nuevos Territorios de Paz y visibilizar cuáles han sido los factores de éxito durante la intervención como aporte estratégico para el diseño de nuevas iniciativas y como insumo para la territorialización de las intervenciones en el marco del posconflicto. En ese sentido, analizaremos los aprendizajes desde tres dimensiones: 1) elementos estratégicos para avanzar de la reactivación económica a la mejora competitiva, 2) aspectos centrales para la implementación del enfoque territorial y 3) aprendizajes relevantes para la participación ciudadana y la gobernanza democrática en regiones posconflicto.

El modelo de concertación planteado para el posconflicto se basa en la concepción de que es posible construir los procesos de desarrollo desde abajo, lo cual se contrapone a modelos centralistas u otros enfoques tradicionales que otorgan un papel protagónico a los actores nacionales o supranacionales, y un rol pasivo y receptivo a los territorios y a sus poblaciones locales. Desde el campo de la antropología se introduce el término development from below, el cual se expresa como un enfoque en contraste con los programas externamente impuestos; el desarrollo desde abajo se focaliza en las necesidades locales, en el rol de las instituciones locales como medio para solventar dichas necesidades y en una percepción local del desarrollo (Pitt, 1976). Este enfoque de participación e intervención fue implementado también en Europa, a través de sus programas Leader y sus iniciativas de Desarrollo Económico Local, y fue integrado a sus instrumentos de cooperación internacional como referente metodológico y conceptual para los programas de desarrollo rural financiados en Colombia. El eje central de estos programas es organizar y movilizar a los actores locales, fomentar procesos participativos de planificación y acuerdo, y generar visiones compartidas de territorio en torno a las cuales articular la acción pública y las estrategias de apoyo e inversión. Son los territorios, a través de una base social organizada y una agenda consensuada de desarrollo, los que adquieren un papel protagónico en la canalización de necesidades y demandas, y en la identificación de los potenciales endógenos y las apuestas competitivas. Este modelo de cocreación de estrategias territoriales y planes de acción permite vincular institucionalidad, academia e iniciativa privada, y se visibiliza como herramienta efectiva para la territorialización de las políticas públicas. En un país como Colombia, donde las vocaciones 
productivas, las dinámicas territoriales y las manifestaciones culturales son tan diversas, las políticas centralistas no han sido capaces de integrar las visiones locales y comunitarias, lo que ha agudizado el distanciamiento entre regiones periféricas y Gobierno nacional, y limitado considerablemente el crecimiento de las apuestas de desarrollo que surgen desde el nivel subregional. El programa NTP, que retoma los aprendizajes acumulados desde los Laboratorios de Paz, asume este marco conceptual y entiende que para generar condiciones de paz territorial es necesario otorgar un papel decisorio a los actores locales, establecer un nuevo diálogo multinivel (que permita integrar las miradas sectoriales en visiones territoriales), y generar capacidades locales para la inclusión socioeconómica y la gobernanza democrática. El desarrollo económico requiere que la participación política y el poder económico se distribuyan, que los frutos del desarrollo se compartan de manera razonablemente equitativa y que haya seguridad para las personas, los grupos sociales y la sociedad. En resumen, el desarrollo requiere gobernanza económica y política (Castañeda, 2017). Este enfoque permite romper de alguna manera con la oposición de ciertos sectores políticos y económicos frente a los procesos redistributivos, los cuales, junto con los impactos multidimensionales del conflicto armado, han ampliado las brechas entre campo y ciudad, y han dificultado el impulso de apuestas económicas alternativas, más acordes con la vocación local y con mayor capacidad de inclusión para las comunidades campesinas.

Con esta lógica de intervención, NTP concentra sus inversiones en cuatro regiones altamente afectadas por el conflicto armado; vincula durante la implementación a entidades públicas, organismos internacionales, sociedad civil y academia, y plantea la siguiente lógica de intervención, la cual guarda muchos paralelismos con la estructuración de los acuerdos, principalmente en lo que se refiere al punto 1 de reforma rural integral, y la formulación e implementación de los PDET (tabla 1).

Durante los próximos meses, el Gobierno nacional y la cooperación internacional centrarán sus acciones en 170 municipios altamente afectados por el conflicto armado, y priorizados para los procesos de transformación integral. En ese sentido, no solo se trata de movilizar recursos y aumentar las inversiones, sino de poner en marcha nuevos enfoques de trabajo con las comunidades, rescatando sus saberes, su experiencia y capacidades, e incorporando a los excombatientes a una vida civil activa. Asílo recoge el Nuevo Acuerdo Final, evidenciando que la paz territorial no puede ser un lema de gobierno sino un nuevo modelo de cocreación de las apuestas de desarrollo y de búsqueda de soluciones con las comunidades. Así queda expresado en el punto 1 en el que se establece que los planes y programas acordados como parte de la RRI deben tener un enfoque territorial, diferencial y de género que implica reconocer y tener en cuenta las necesidades, características y particularidades económicas, culturales y sociales de los territorios, de las mujeres en todo su ciclo vital, de las comunidades rurales y de grupos en condiciones de vulnerabilidad, garantizando la sostenibilidad socioambiental (Gobierno Nacional de Colombia, 2016). Más allá de la conceptualización o del enfoque teórico que se aborda desde la negociación, el gran 
TABLA 1. INVERSIONES DEL PROGRAMA NUEVOS TERRITORIOS DE PAZ EN CUATRO REGIONES AFECTADAS POR EL CONFLICTO ARMADO

\begin{tabular}{|c|c|c|c|c|c|}
\hline & & $\begin{array}{l}\text { Dpto. } \\
\text { Guaviare }\end{array}$ & $\begin{array}{l}\text { Dpto. } \\
\text { Caquetá }\end{array}$ & $\begin{array}{c}\text { Bajo } \\
\text { Magdalena }\end{array}$ & $\begin{array}{l}\text { Canal del } \\
\text { Dique }\end{array}$ \\
\hline \multirow{2}{*}{$\begin{array}{l}\text { Inclusión } \\
\text { socioeconómica }\end{array}$} & $\begin{array}{l}\text { Promoción de modelos productivos } \\
\text { sostenibles }\end{array}$ & \multirow{11}{*}{$7.473 .166 €$} & \multirow{11}{*}{$6.751 .721 €$} & \multirow{11}{*}{$7.909 .544 €$} & \multirow{11}{*}{$7.541 .453 €$} \\
\hline & Mejora de la seguridad alimentaria & & & & \\
\hline \multirow{2}{*}{$\begin{array}{l}\text { Tierras y } \\
\text { territorio }\end{array}$} & $\begin{array}{l}\text { Formalización de la propiedad y } \\
\text { procesos de restitución }\end{array}$ & & & & \\
\hline & $\begin{array}{l}\text { Actualización catastral y capacidades } \\
\text { para la planificación }\end{array}$ & & & & \\
\hline \multirow{3}{*}{$\begin{array}{l}\text { Generación de } \\
\text { capacidades }\end{array}$} & $\begin{array}{l}\text { Fortalecimiento institucional y } \\
\text { gobernanza democrática }\end{array}$ & & & & \\
\hline & $\begin{array}{l}\text { Participación ciudadana e incidencia } \\
\text { política }\end{array}$ & & & & \\
\hline & $\begin{array}{l}\text { Fortalecimiento de organizaciones } \\
\text { locales }\end{array}$ & & & & \\
\hline \multirow{4}{*}{$\begin{array}{l}\text { Enfoques } \\
\text { transversales }\end{array}$} & Inclusión de género & & & & \\
\hline & Enfoque diferencial y étnico & & & & \\
\hline & Construcción de paz & & & & \\
\hline & Conservación del medio ambiente & & & & \\
\hline
\end{tabular}

Fuente: elaboración propia.

reto es cómo instrumentalizar y operativizar en campo ese nuevo enfoque, y cuáles son los factores estratégicos en los procesos de territorialización, inclusión efectiva de los sectores vulnerables y transformación de las dinámicas económicas, políticas y sociales en las regiones históricamente afectadas por la presencia de actores armados, y en muchos casos, por una cotidianización de la ilegalidad y las acciones al margen de la ley y la institucionalidad. "Durante todas estas décadas, el campo colombiano no ha contado con las suficientes políticas de desarrollo rural y social capaces de compensar los sesgos provenientes de las políticas comerciales y de apoyo a la producción [...] en el modelo general de desarrollo del país, la agricultura ha sido esencialmente funcional a las necesidades de crecimiento de los sectores urbanos e industrial, además, dicho modelo incorpora una visión esencialmente productivista de las actividades agrícolas" (DNP, 2007).

Con el proceso de desarme y dejación de armas ya concluido, y en lo que podría considerarse el punto de partida de las inversiones del posconflicto, la experiencia práctica de NTP puede darnos algunas luces sobre estrategias, enfoques o factores clave para la puesta en práctica del enfoque territorial, la dinamización 
económica y el restablecimiento del diálogo, y la construcción de apuestas de desarrollo desde los sectores vulnerables y las comunidades históricamente excluidas. A continuación, y de manera sintética, presentamos algunas de las ideas fuerza que consideramos más relevantes para el diseño de programas y para la implementación de los acuerdos desde el ámbito comunitario, local y subregional.

\section{ASPECTOS CENTRALES PARA LA IMPLEMENTACIÓN DEL ENFOQUE TERRITORIAL}

1. En primer lugar, es necesario transitar de un enfoque exclusivamente sectorial, que ha primado en las estrategias de gobierno y en los programas de cooperación internacional, a un enfoque territorial que, de manera obligatoria, parte de procesos participativos e intersectoriales de planificación.

Este componente no se basa exclusivamente en la elaboración de agendas sino que debe centrarse en el establecimiento de consensos.

Hoy es casi un lugar común hablar en el país de desarrollo rural territorial, o con enfoque territorial, como lo denominamos. No obstante, no siempre es claro a lo que nos estamos refiriendo, ni es obvio que tengamos acuerdos en lo que por el entendemos: ¿qué es lo que queremos hacer y lograr los colombianos cuando hablamos de desarrollo rural con enfoque territorial? ¿Cómo lo debemos construir? ¿Cuál es la institucionalidad que para ello requerimos, tanto en el ámbito nacional como en el territorial, tanto en lo gubernamental como en lo no gubernamental? ¿Qué papel deben jugar las instituciones gremiales y empresariales y las organizaciones sociales y de la sociedad civil en este desarrollo rural con enfoque territorial? (Perry, 2014).

Los territorios afectados por el conflicto armado han desarrollado modelos de relacionamiento antagónicos entre comunidades e institucionalidad, y diferencias profundas entre los actores territoriales, económicos y sociales, cuya forma de entender el modelo de desarrollo, la proyección del territorio y las causas del conflicto les han enfrentado históricamente. En ese sentido, un primer punto será el restablecimiento del diálogo, la puesta en marcha de ejercicios de reflexión y debate a nivel intersectorial, y la búsqueda de acuerdos de mínimos que permitan posicionar la agenda del territorio ante la ayuda internacional y la inversión pública. El hecho de poner en marcha nuevos espacios de decisión o fortalecer los ya existentes, dentro de un enfoque participativo, no es suficiente para garantizar una correcta articulación y alineación entre política pública, actores locales y comunidad. De manera paralela, es importante realizar un análisis y un debate a nivel territorial, que nos permita concertar un marco o una apuesta concreta para el desarrollo del territorio. El ejercicio combinará el análisis de los diagnósticos territoriales existentes con la puesta en marcha de un diálogo abierto, en el cual todos los actores económicos y sociales puedan expresar sus intereses, limitaciones y potencialidades (Unión Europea, Prosperidad Social y Red Adelco, 2016). 
2. La sostenibilidad de las visiones compartidas de región y de las apuestas endógenas de desarrollo dependerá de la capacidad de crear y consolidar redes territoriales de trabajo.

Uno de los principales problemas que enfrentan las agendas territoriales es su dependencia frente a los procesos políticos. Una vez los actores locales, con papel protagónico de la sociedad civil organizada y los sectores vulnerables, alcanzan ciertos consensos sobre la proyección y las apuestas del territorio, es necesario establecer modelos de gobernanza que permitan posicionar la agenda a través de iniciativas de participación ciudadana e incidencia política. Se trata de que las políticas locales y las estrategias de desarrollo se articulen a la agenda, y no viceversa, ya que esta expresa las demandas y las expectativas de las comunidades, y servirá como eje articulador de las acciones y las inversiones. La gran debilidad son los modelos de gobernanza de estas agendas, que deben recaer y defenderse a través de los actores locales, muchas veces incapacitados por la falta de recursos o las dificultades de acceso a los espacios de toma de decisión. El proceso de posconflicto podría concentrar esfuerzos en la conformación y el funcionamiento de estas estructuras territoriales, las cuales serán sostenibles en la medida en que puedan incidir de manera efectiva en la toma de decisiones, favorecer el intercambio de conocimiento, y vincularse a los procesos de planificación, ejecución, seguimiento y evaluación. Estas nuevas visiones de gobernanza regional deberían incorporar y alinearse con las siguientes cuestiones, ¿en qué medida las instituciones de la gobernanza regional pueden constituirse en el motor de un proceso de desarrollo más reflexivo, democrático, armónico y equitativo a partir de potenciar las relaciones entre los actores regionales? ¿Cuál es el rol reservado a la gobernanza regional en las discusiones sobre implementación de políticas? (Vigil y Fernández, 2012). Nuevos Territorios de Paz las aborda desde la práctica y concluye que es a través del fortalecimiento de estas redes intersectoriales de trabajo, expresadas en espacios formales e informales de participación y decisión, las que pueden liderar y mantener las apuestas territoriales en el medio y largo plazo.

\section{En las apuestas territoriales de desarrollo debe estar presente la empresa privada, mu- chas veces invisibilizada por los programas de cooperación, no tanto a través de sus iniciativas de responsabilidad social empre- sarial sino como generadora de oportunida- des, conectora de comunidades y mercados, y difusora de conocimiento estratégico para el territorio.}

Quizás una de las principales debilidades que se han evidenciado en los procesos de fortalecimiento organizacional y desarrollo rural ha sido la incapacidad de vincular a las empresas privadas del territorio en el fortalecimiento de los modelos de negocio y el cierre de brechas entre oferta y mercados formales a nivel local. No podemos entender los modelos de desarrollo territorial sin vincular a las empresas privadas (no solo organizaciones de base y pymes, que suelen ser el sujeto de atención, sino también empresas de mayor tamaño que puedan servir como aliados ancla que faciliten los procesos de empresarización, proveeduría y desarrollo comercial). Es muy pertinente 
incluir nuevas reflexiones respecto a la relación entre empresa privada y posconflicto, donde el sector privado reconoce su rol y responsabilidad, pero desconoce las formas para canalizar su esfuerzo, teme que esa responsabilidad la termine asumiendo en su totalidad, cree que el Gobierno envía mensajes contradictorios o divergentes, le preocupa que con estos procesos se premie a las personas que han estado al margen de la ley, percibe que el Gobierno solo lo tiene en cuenta cuando enfrenta problemas financieros y siente que no se le explica lo suficiente sobre cómo se invierten los recursos aportados. Para generar confianza y canalizar los esfuerzos del sector privado, el Estado ha elaborado una serie de mecanismos que permiten desarrollar acciones específicas, como generación de empleo directo, identificación y asesoría a proyectos productivos, apadrinamiento de pymes, generación de pasantías o prácticas laborales, compra de productos o servicios provistos por beneficiarios del programa, implementación de proyectos productivos, etc. (González, 2016). De acuerdo con los aprendizajes de NTP en estos seis años se concluye que, en primer lugar, la empresa debe estar presente en los procesos de planificación y diseño de programas. Su conocimiento de las cadenas productivas, de sus limitaciones y potencialidades, y su visión sobre la rentabilidad y la sostenibilidad de los negocios puede generar mayor eficacia y eficiencia en las inversiones. En segundo lugar, pueden tener un papel protagónico en el fortalecimiento de proveedores y el desarrollo de prestadores de servicios desde lo local, en la transferencia de información estratégica a las comunidades productoras y sus organizaciones de base (sobre requerimiento de los mercados y caracterización de la demanda), y en el acercamiento de asistencia técnica especializada y acompañamiento práctico a las organizaciones más incipientes. Las apuestas locales de desarrollo en el marco del posconflicto deberían incorporar enfoques de crecimiento inclusivo, de consolidación de cadenas de valor y de procesos de producción y comercialización desde el ámbito regional.

\section{La construcción de agendas territoriales, el establecimiento de consensos a nivel in- tersectorial y la participación activa de los actores locales en los procesos de desarrollo debería materializarse paralelamente en po- líticas públicas pertinentes, que integren el enfoque territorial.}

Otro de los grandes retos que ha identificado el programa Nuevos Territorios de Paz es la necesidad de trasladar los consensos y las agendas territoriales a los instrumentos formales de planificación, es decir, integrar esos acuerdos intersectoriales en los planes municipales de desarrollo, los planes de ordenamiento territorial (РОт) y en los programas locales de inversión. El principio de alineación que propone la Organización para la Cooperación y el Desarrollo Económicos (OCDE) en la Declaración de París sobre la eficacia de la ayuda establece la necesidad de que los recursos de cooperación estén alineados a las políticas públicas. Este proceso de construcción también puede ser de abajo hacia arriba, donde las agendas consensuadas a nivel local son materializadas en políticas públicas territoriales y en los bancos municipales de proyectos, los cuales se convierten en herramientas articuladoras de los programas y las inversiones. Esta propuesta de regionalización ha sido promovida en el 
último Plan Nacional de Desarrollo y en el documento "Regionalización Plan Plurianual de Inversiones 2015-2018”, del Departamento Nacional de Planeación (DNP), y será uno de los ejes articuladores para los procesos de formulación e intervención en el marco del posconflicto. El reto que desde NTP se ha venido visibilizando y pilotando, es cómo expresar el enfoque diferencial y de subregionalización en los instrumentos locales de planificación, más cercanos a las comunidades y a su realidad productiva y socioeconómica.

\section{Promover modelos eficientes y sostenibles de desarrollo territorial, con capacidad para la inclusión socioeconómica y reducción de conflictividades, exige orientar esfuer- zos técnicos y financieros a los procesos de ordenamiento territorial, catastro rural y formalización de la propiedad.}

Las apuestas territoriales de desarrollo, bajo criterios de inclusión, tendrían que ser respetuosas con las vocaciones productivas de las comunidades y, bajo criterios de sostenibilidad, garantizar las posibilidades de mercado y las condiciones de competitividad. Existe una estrecha relación entre desarrollo económico sostenible y gestión del territorio, y esta relación se agudiza en las regiones más afectadas por la guerra, donde las mayores conflictividades giran en torno al acceso y uso de la tierra. Recogemos las conclusiones de Felipe Guzmán, quien aborda la temática del catastro multipropósito y las problemáticas de tierras, y su impacto directo en la reforma rural integral, y hace énfasis en que esta política pública debe aprovechar el conocimiento geográfico, agrológico y ambiental acumulado sobre el territorio y ponerlo al servicio de la población campesina y de los productores eficientes, para proteger la sostenibilidad de los recursos y ordenar mejor la distribución de la población campesina en el territorio más productivo. Esta política pública puede ser el insumo fundamental para el diseño e implementación de acciones que busquen justicia distributiva, transferencia tecnológica y desarrollo rural al campo colombiano (Guzmán, 2017). En ese sentido, y de acuerdo con la experiencia de NTP, que desarrolló todo un resultado de "Tierras y Territorio", es necesario centrar los esfuerzos en tres elementos clave. En primer lugar, las instituciones locales y las comunidades deben entender la importancia del ordenamiento territorial (estrategias de sensibilización e incentivación), y es necesario mejorar las capacidades locales para los procesos de planificación, actualización y gobernanza de los рот. En segundo lugar, hay una estrecha relación entre las iniciativas de reactivación económica y los procesos de actualización catastral y formalización. Este enfoque se ha recogido de manera explícita en los acuerdos pero debe ser integrado también en las estrategias de mejora competitiva y en la planificación de dichas apuestas, priorizando aquellas comunidades más afectadas por la inseguridad jurídica y la desinversión. Por último, se ha identificado la alta vulnerabilidad de las comunidades indígenas y afrodescendientes (con visiones propias y tenencia colectiva) para posicionar e integrar sus planes de vida en los instrumentos de ordenamiento territorial. La falta de participación de los grupos étnicos en los procesos de participación impide posicionar sus propuestas de desarrollo e integrarlas de manera armónica en los planes de desarrollo. No podemos entender el desarrollo territorial 
sostenible y la reducción de conflictividades sin la inclusión efectiva y respetuosa de sus planes de vida. Este último punto demanda esfuerzos complementarios por parte de la institucionalidad y los actores de desarrollo.

\section{La generación de capacidades locales, a nivel institucional y organizacional, debe ser un elemento transversal en los programas de desarrollo con enfoque territorial.}

Tanto en las estrategias de desarrollo económico local como en los principios planteados por la Misión Rural y la reforma rural integral, los actores territoriales deben tener un papel protagónico en los procesos de planificación, ejecución, seguimiento y gobernanza. Teniendo en cuenta el alto grado de exclusión y vulnerabilidad de muchas comunidades, los procesos de formación, acompañamiento práctico y transferencia de conocimiento son fundamentales como estrategia de sostenibilidad. A pesar de que muchas veces hay una visión escéptica frente a la efectividad de estas inversiones, principalmente por su falta de resultados directos, no es posible generar autonomía y liderazgo territorial si no existen capacidades en la sociedad civil organizada y en la institucionalidad local para participar de manera cualificada y liderar los procesos de manera autónoma una vez concluida la financiación externa. Hay que tener en cuenta que el desarrollo territorial depende en gran medida de liderazgos. Para ello, es muy importante intercambiar información entre cooperantes y entidades públicas, y dar continuidad a los procesos de acompañamiento y formación a los actores territoriales. Las juntas de acción comunal, las alcaldías municipales, los espacios locales de participación (formales e informales), los gremios y las organizaciones de base, sociales y productivas, se identifican como las plataformas más efectivas para liderar, gobernar e impulsar las apuestas territoriales de desarrollo.

\section{ELEMENTOS ESTRATÉGICOS PARA AVANZAR DE LA REACTIVACIÓN ECONÓMICA A LA MEJORA COMPETITIVA}

Las regiones priorizadas en el marco del posconflicto, históricamente afectadas por el enfrentamiento armado y el abandono estatal, han visto limitado el crecimiento de sus economías locales. No podemos hablar de paz territorial si no se mejoran los modelos de negocio de las familias campesinas, se crean oportunidades de empleo e ingresos desde la ruralidad, y se avanza en la modernización de sus iniciativas productivas y comerciales, conectando apuestas comunitarias y mercados. Los casos de proyectos productivos o de generación de ingresos de comunidades rurales pobres $y$ de emprendimientos empresariales exitosos son relativamente escasos. La política gubernamental en esta materia ha tenido numerosos fracasos. Si bien es cierto algunos programas pueden haber contribuido a potenciar las capacidades productivas de productores medianos $\mathrm{y}$ de algunos pequeños que ya contaban con una capacidad productiva básica, ellos no parecen haber llegado de manera significativa a la población pobre y pobre extrema (Perry, 2010). Hay que tener en cuenta que en el corto plazo la cooperación internacional y la inversión pública pueden ayudar a la reactivación económica, pero en el mediano y largo plazo debe ser el mercado, y en menos medida el Estado, 
el que garantice la sostenibilidad. Para ello es necesario unir vocaciones productivas y potenciales endógenos con inversiones estratégicas y mercados. A continuación, destacamos seis aprendizajes al respecto.

\section{Uno de los elementos clave para la reduc- ción de las desigualdades y la generación de oportunidades desde la ruralidad es la pro- moción de modelos productivos sostenibles.}

De acuerdo con el enfoque de desarrollo económico local, estos modelos deberían alinearse con la vocación productiva y el contexto cultural de los territorios, pero respetando criterios de sostenibilidad ambiental, inclusión social y competitividad (articulación con la demanda). Para ello, se parte de ejercicios de planificación donde las comunidades identifican sus potenciales endógenos, visibilizan sus capacidades locales y toman decisiones de acuerdo con una visión de mercado (local, regional, nacional e, incluso, internacional). En el marco del programa se apoyaron más de 1.600 familias y uno de los principales aprendizajes fue que las comunidades dan mayor valor, en muchos casos, a la transferencia metodológica, la formación y la asistencia técnica que al apoyo financiero. Las comunidades no cuentan con acceso a una oferta formativa pertinente y este es uno de los elementos más valorados. Esto quiere decir que muchas veces la cooperación técnica es más efectiva que la cooperación financiera, y que el acompañamiento práctico, la asesoría individualizada y los ejercicios demostrativos, vinculados a procesos de réplica, son unas de las estrategias de mayor impacto.

\section{En el territorio conviven diferentes tipos} de economías y es fundamental facilitar la interacción, los encadenamientos y las alianzas entre estas. Hay una relación estrecha entre paz territorial y diálogo de modelos económicos locales.

Así lo recogía la Misión para la Transformación del Campo, al afirmar que lo rural es estratégico para el desarrollo, no subsidiario y no solamente por razones económicas sino también por la necesidad de superar los factores históricos del conflicto y construir la paz. Esta paz parte de entender el campo como un espacio donde caben y pueden convivir armoniosamente los grandes, los medianos y los pequeños agricultores en conjunto con actores de otras actividades económicas (Departamento Nacional de Planeación, 2014). Avanzar hacia modelos de competitividad territorial inclusivos requiere la búsqueda de consensos entre los actores económicos. Esto se expresa con claridad en los territorios con grandes problemáticas sobre la tierra y en mayor medida en aquellos que se resisten a los modelos económicos extractivos y a la gran inversión multinacional. En el caso de NTP esto se ha realizado a través de espacios intersectoriales de participación como, por ejemplo, la "Cámara Intergremial de Caquetá”, la cual integra a las diferentes apuestas productivas, representando las cadenas de mayor fortaleza, pero también las más incipientes, y generando consensos territoriales para los procesos de inversión, elaboración de agendas y búsqueda de alianzas locales. Este puede convertirse en un espacio interesante de articulación para las entidades 
públicas y la cooperación internacional, y en una plataforma con capacidad de incidencia, que ponga encima de la mesa las conflictividades entre las diferentes visiones de desarrollo de los agentes económicos y de los modelos de negocio que estos lideran.

\section{La reforma rural integral destaca el papel protagónico de los actores locales. Las orga- nizaciones de base, con el debido acompa- namiento, pueden ser ejecutoras directas de las inversiones y estas pueden ser una de las estrategias más efectivas de fortalecimiento y generación de capacidades.}

Para hacer operativo este enfoque, Nuevos Territorios de Paz puso en marcha la herramienta "Apoyo financiero a terceros" (AFT). Durante el programa, más de 220 asociaciones locales pudieron formular proyectos de fortalecimiento productivo y comercial, y desarrollo comunitario, y operaron de manera directa más de 10.000 millones de pesos (3 mill. €). Este instrumento permite apoyar las apuestas endógenas de las comunidades y avanzar en ejercicios de fortalecimiento organizacional desde la práctica. Es necesario brindar acompañamiento, principalmente a los procesos administrativos y contables, pero los aprendizajes son de gran impacto. Las organizaciones asumen todas las competencias durante la ejecución y, desde la práctica, adelantan ejercicios de planificación estratégica, priorización de inversiones, rendición de cuentas y gestión transparente. Una vez concluidos los proyectos no solo cuentan con capacidad para gestionar de manera autónoma sus modelos de negocio sino que han logrado consolidar procedi- mientos internos, ejercicios de formalización empresarial y manejo responsable de recursos. Los PDET, en su fase de diseño e inversión, establecen el papel protagónico de las juntas de acción comunal y las organizaciones locales en el proceso de implementación, y afirman que la participación debe estar presente en todas las fases de elaboración para garantizar su legitimidad (DNP, Alta Consejería para el Posconflicto y Alto Comisionado para la Paz, 2016). En ese sentido, la figura de AFT, con sus dimensiones de operación directa y fortalecimiento práctico, puede servir como estrategia de referencia para la implementación de los acuerdos, la generación de confianza y capacidades, y la capitalización temprana de los dividendos de la paz.

\section{Es importante revalorizar los modelos productivos locales, vincular institutos científicos y academia a los procesos de tec- nificación y generación de valor agregado, y motivar las redes territoriales de conoci- miento.}

La sostenibilidad de las apuestas productivas y comerciales dependerá en gran medida del relacionamiento y las alianzas entre actores territoriales, y de la capacidad de generar e intercambiar conocimiento innovador y pertinente para la dinamización de sus actividades productivas. De la experiencia de NTP podemos destacar tres elementos clave. En primer lugar, identificar y revalorizar las buenas prácticas al interior de los territorios y facilitar la difusión de estas entre pares. En el marco del programa se han desarrollado más de 25 giras técnicas entre pobladores locales 
y experiencias exitosas, las cuales se han posicionado como una de las estrategias más efectivas para la sensibilización, la promoción de buenas prácticas y la multiplicación de aprendizajes. En segundo lugar, es fundamental la participación de la academia y los institutos científicos en las iniciativas de desarrollo rural y mejora competitiva. Estas entidades tienen la capacidad de realizar investigación aplicada, documentar y estandarizar los modelos locales de producción y generar información estratégica para su fortalecimiento. Por último, uno de los grandes retos identificados es cómo generar plataformas sectoriales y territoriales de trabajo, vinculando sectores productivos, academia e institucionalidad, que permitan democratizar estos aprendizajes, transferirlos a las entidades territoriales y los programas de desarrollo y compartirlos con la base productiva y las comunidades más aisladas. Esto se recoge de manera explícita en los acuerdos de paz, y será un eje fundamental en los procesos de tecnificación, innovación y generación de valor agregado, vinculando asistencia técnica y tecnológica con los resultados de procesos de investigación e innovación agropecuaria, incluido el uso de las tecnologías de la comunicación y la información (Gobierno Nacional de Colombia, 2016).

\section{Una de las grandes debilidades es la des- conexión entre comunidades productoras y mercados, y las dificultades para establecer diálogos y alianzas entre ellos.}

Los proyectos de desarrollo no han logrado superar el enfoque productivista y esto pone en riesgo la continuidad y la rentabilidad de las estrategias. Como mencionábamos, los proyectos de inversión permiten reactivar las economías locales en el corto plazo, pero si no hay un relevo y un relacionamiento con el mercado, no habrá sostenibilidad en el mediano y largo plazo. Podemos decir que este es uno de los grandes cuellos de botella en los procesos de desarrollo rural y en las iniciativas de generación de ingresos. En el marco de NTP se han apoyado más de 450 organizaciones de base, mediante procesos de formación, asistencia técnica y apoyo financiero, pero solo una minoría de ellas han logrado afianzar alianzas comerciales de largo aliento. Esto era uno de los elementos clave identificados por la Misión Rural para la consolidación de los modelos de negocio, donde se afirmaba que las asociaciones de productores pueden jugar un papel fundamental en la comercialización de los productos, incluida la comercialización internacional, así como en la formación de agroindustrias o entidades de servicios de distinta escala, con un enfoque de cadenas productivas (Departamento Nacional de Planeación, 2014). No podemos hablar de generación de valor agregado o mejora competitiva sin afrontar algunos de los cuellos de botella que las organizaciones de base presentan en la actualidad. En ese sentido, identificamos dos elementos importantes que deberían ser tenidos en cuenta en los programas de desarrollo rural y empresarización en el posconflicto. Por un lado, destinar recursos al inicio de los proyectos, de manera previa a la capitalización de las organizaciones y la definición de sus modelos de negocio, que permitan acercar base productiva y posibles clientes (institucionales y privados). Esto permitirá conocer los poten- 
ciales de venta, los requerimientos y priorizar inversiones que permitan cerrar brechas entre oferta y demanda a nivel local, sobre todo en términos de calidad, certificación, presentación y adecuación de producto. Por otro lado, vincular a las empresas locales, los gremios y los potenciales socios comerciales en los ejercicios de asistencia técnica y acompañamiento. Muchas veces la carga formativa y de transferencia metodológica se centra en los equipos técnicos de los proyectos, pero puede ser muy efectivo ceder competencias y contenidos a los privados, por su conocimiento del mercado y su potencialidad como clientes. Esto permitirá establecer alianzas, dar especificidad a los procesos de formación y generar relaciones que perduren más allá de la ejecución del proyecto.

\section{Debemos entender la competitividad des- de sus diferentes niveles y en su relación con el territorio. Ser competitivo no quiere decir, en muchos casos, ser capaces de acceder a los mercados nacionales o internacionales, sino tener capacidades para generar modelos de negocio inclusivos y sostenibles, capaces de crear empleos e ingresos estables para las poblaciones locales.}

Al hablar de competitividad muchas veces se excluye el componente social, pero en el marco del posconflicto son elementos interdependientes, estrechamente relacionados. Ya se había identificado en el ańo 2013 en un documento de lineamientos de política pública elaborado por DNP, y se espera avanzar en los próximos años en esa dirección, en el marco de la política nacional de competitividad que lidera el Ministerio de Industria, Comercio y
Turismo con el apoyo de Unión Europea. Lo que se plantea con estas intervenciones es poder avanzar hacia procesos de competitividad territorial, la cual va más allá del concepto de competitividad porque se sustenta no solo en términos económicos sino de redistribución entre los actores territoriales, así como mayor responsabilidad social y con el medio ambiente. Es decir, que un territorio adquiere carácter competitivo si puede afrontar la competencia del mercado y garantizar al mismo tiempo la viabilidad medioambiental, económica, social y cultural, aplicando lógicas de red y de articulación interterritorial (Departamento Nacional de Planeación, 2013). En ese sentido, y de acuerdo con la experiencia de NTP, se identifica que en muchos casos (principalmente en las zonas rurales dispersas o de difícil acceso) las intervenciones se deben centrar en el relacionamiento entre oferta y demanda local, y en la optimización de las intermediaciones. Esto puede tener grandes impactos en términos de ingresos y de sostenibilidad empresarial. En otros casos, es posible acceder a mercados regionales y para ello es importante avanzar en planes de abastecimiento territorial y en estrategias de comercialización como los mercados campesinos, que vinculan comunidades productoras y cliente final. Por último, algunas apuestas productivas, como el café, el cacao u otros productos de alto valor agregado, pueden focalizar mercados nacionales e internacionales para lo cual es fundamental apoyar los procesos de adecuación de producto, participación en ruedas de negocio y ferias, o fomento a la conformación de figuras comerciales bajo esquemas de asociatividad. 


\section{APRENDIZAJES RELEVANTES PARA LA PARTICIPACIÓN CIUDADANA Y LA GOBERNANZA DEMOCRÁTICA EN REGIONES POSCONFLICTO}

La construcción de paz territorial, más allá de la necesidad de territorializar los acuerdos de paz y las políticas públicas de manera acorde a las especifidades regionales, exige nuevos modelos de diálogo social, participación política y expansión de los principios democráticos. Si la construcción de paz se sustenta en la posibilidad de transformar los conflictos sobre la base de redes de relaciones entre personas y grupos, en la generación de plataformas para la interacción, en la capacidad de dar forma a un entorno de confianza, y en la posibilidad de contar con instituciones que favorezcan la acción colectiva, la participación ciudadana se constituye en un elemento central para dichos propósitos en tanto patrón de relacionamiento basado en el diálogo, un escenario de interacción con potencial para fomentar la cooperación y la coordinación, y un mecanismo para transformar pacíficamente la realidad social (Maldonado, 2016). El programa Nuevos Territorios de Paz, de manera paralela a la generación de capacidades locales, ha centrado gran parte de su intervención en el acercamiento entre institucionalidad local y comunidades. Estas actividades se realizan en doble vía, mejorando el funcionamiento de la institucionalidad local y promoviendo la participación cualificada y pertinente de las comunidades rurales y vulnerables. Aunque este componente no ocupó un punto exclusivo de los diálogos de La Habana, es transversal a toda la implementación y será eje central en la formulación e implementación de los PDET, en las iniciativas de sustitución de cultivos ilícitos y en la restauración de condiciones pacíficas de convivencia. A continuación se presentan algunas ideas fuerza para su ejecución.

\section{Es fundamental fortalecer los espacios} locales de participación, pero las acciones no se pueden limitar a incluir de manera testimonial a los sectores vulnerables o a las poblaciones históricamente excluidas, sino que estas deben ser incluidas de manera efectiva en la toma de decisiones.

Este es uno de los grandes retos en el marco del posconflicto. En el marco de NTP se ha acompańado técnicamente a más de 50 municipios en sus procesos de planificación y se han establecido planes de fortalecimiento para sus espacios locales de participación. Se han evidenciado impactos positivos en el acercamiento entre comunidades e institucionalidad, y se ha avanzado en el proceso de legitimación de lo público, pero a la vez se ha identificado que, en la mayoría de los casos, la participación de las poblaciones vulnerables (campesinas, étnicas, etc.) no ha tenido los impactos esperados en el diseño de las políticas públicas y en la orientación de los recursos. Esto puede ser contraproducente y revertir la generación de confianza. En ese sentido, se identifica como necesario acompañar los procesos de fortalecimiento institucional con ejercicios de sensibilización y monitoreo desde el Gobierno nacional. Es importante visibilizar la importancia de realizar ejercicios participativos de planificación, mostrar los efectos positivos en términos de legitimidad y transparencia, e incluso desarro- 
llar incentivos que motiven a los municipios a evitar procesos estandarizados de planeación, canalizando de manera efectiva las demandas de los ciudadanos y materializándolas en los instrumentos de política y, por tanto, en sus inversiones. Las entidades territoriales asumen estos compromisos como un mero trámite o un procedimiento de obligado cumplimiento, por lo que es necesario articular planes de fortalecimiento con modelos de seguimiento e incentivo desde la centralidad. La experiencia en Guaviare, que hizo especial hincapié en el trabajo con comunidades indígenas, concluye que a lo largo de la intervención se evidenció que a pesar de que existen espacios de participación, como los CMDR, no son apropiados por los campesinos y los indígenas.

Muy pocos conocían su existencia, y se reconocía que las comunidades se sentían más identificadas con las Juntas de Acción Comunal de cada vereda. Es importante detenerse en este aspecto, ya que este tipo de espacios fueron creados para una interacción directa entre la comunidad y los gobernantes, donde de manera conjunta se identifiquen las necesidades y se propongan soluciones. Sin embargo, estos escenarios los ha convertido la institucionalidad en espacios de rendición de cuentas (Hilfswerk Austria, Unión Europea y Prosperidad Social, 2017).

La participación de las comunidades históricamente excluidas será sostenible en la medida en que haya influencia real en la toma de decisiones, en la orientación de recursos o en el diseño de políticas públicas locales, y el proceso de posconflicto. La formulación de los PDET es una oportunidad significativa para impulsar estos nuevos modelos de relacionamiento.
2. No podemos hablar de gobernanza democrática y paz territorial sin procesos de interculturalidad. Muchos de los territorios posconflicto tienen grandes problemas de exclusión de las comunidades étnicas, procesos de colonización no resueltos y grandes conflictividades en torno a los grupos poblacionales, que dificultan la convivencia pacífica y el desarrollo sostenible.

Nuevos Territorios de Paz ha desarrollado estrategias de trabajo con las comunidades indígenas, pero vinculadas a procesos de diálogo en lo local y de relacionamiento y acuerdo con los diferentes actores y sectores poblacionales. Se trata de equilibrar el enfoque diferencial con el enfoque territorial. Uno de los aspectos más relevantes en la implementación de los acuerdos de paz y que, de alguna manera, ha quedado un poco invisibilizado en su presentación ha sido el papel protagónico que deben jugar las minorías étnicas (afrodescendientes, indígenas, etc.) en los procesos de participación, planificación e inversión en el territorio. Debemos tener en cuenta que muchas de estas comunidades se encuentran en zonas priorizadas para el posconflicto, donde ha habido una fuerte presencia de actores armados y donde se evidencian fuertes impactos en términos de ruptura del capital social, desplazamiento forzado, destrucción de medios de vida tradicionales e influencia de las economías ilícitas. Con base en las experiencias del programa destacamos tres aprendizajes clave. En primer lugar, es fundamental incluir iniciativas de interculturalidad y diálogo entre comunidades campesinas, muchas veces colonos, $\mathrm{y}$ comunidades indígenas. Esto contribuirá a 
generar confianza, intercambiar conocimiento y establecer compromisos para los procesos de retorno, reasentamiento y fortalecimiento comunitario. Asimismo, será fundamental para reducir las conflictividades existentes. En segundo lugar, promover programas y proyectos con las comunidades indígenas, de acuerdo con sus propias visiones de desarrollo y sus apuestas productivas, culturales, sociales e identitarias. Muchas veces se relega a los expertos una serie de actividades que se pueden realizar con las comunidades y con los conocimientos y prácticas que ya tienen. Por ejemplo, la autonomía desde un punto de vista integral: alimentaria (que se puede realizar a través de las chagras y los conocimientos tradicionales), médica (que se puede realizar revalorizando los conocimientos ancestrales) u organizativa (que tiene un proceso orgánico de conformación, por lo que no se debe forzar a que se unan sobre una identidad común, todavía no consolidada, dado que existen diferencias considerables entre los distintos grupos étnicos). La autonomía se debe trabajar con las familias por pueblo indígena. Unir a las comunidades que, a pesar de estar separadas por diferentes razones históricas, tienen puntos en común y que pueden encontrar nexos identitarios y culturales. Se trata de promover el acercamiento respetando las diferencias, intentando generar ciertos mínimos comunes entre los pueblos para los procesos de participación e incidencia. Para ello, es importante destacar las metodologías desarrolladas en el Guaviare, que plantean espacios de encuentro entre diversas comunidades y una metodología de trabajo basada en tres etapas: conocernos, reconocernos y establecer acuerdos de convivencia (Hilfswerk Austria, Unión
Europea y Prosperidad Social, 2017). Por último, trabajar con las autoridades indígenas el tema de autonomía, es decir, que un resguardo indígena es una entidad territorial que está a la altura de una administración municipal. Los proyectos deben tener en cuenta que las autoridades indígenas tienen un nivel de decisión y de planificación por encima de los espacios formales de un municipio, y eso debería ser uno de los criterios de trabajo para próximas iniciativas. En el marco de otros proyectos se recomienda trabajar de manera paralela en dos componentes: a través del decreto autónomo de las comunidades indígenas (que les genere capacidades para gestionar sus recursos de manera directa, y dar efectividad al Decreto 1953 del 2014), al tiempo que se trabaja con los espacios formales (CMDR y otros), y establecer una relación más directa entre municipio y autoridades indígenas.

3. Para poder vincular a los sectores vulnerables y a las organizaciones de base en los procesos de participación, incidencia política y veeduría ciudadana es importante compatibilizar cuatro niveles de trabajo: generar formas de ingresos que permitan la participación, elaborar agendas organizacionales y escalarlas a nivel comunitario, brindar formación estratégica para la participación cualificada y facilitar el acceso a los espacios institucionales.

La nueva estructura institucional y de construcción de política pública que se pone en marcha en el posconflicto requiere una sociedad civil organizada, con capacidad para planificar sus acciones, canalizar sus demandas 
e incidir de manera efectiva en los espacios de decisión. Una de las conclusiones de NTP es que lo productivo, lo político y lo organizativo, juntos, construyen una visión compartida del territorio que puede derivar en modelos propios de desarrollo, en una reducción de las conflictividades y, en última instancia, en la consolidación de paz territorial. Bajo esa lógica de intervención, una de las primeras actividades es generar medios de vida sostenibles que permitan a las familias productoras superar las economías de subsistencia y destinar esfuerzos y tiempo a los procesos de organización local, participación ciudadana e incidencia. Pero no basta con generar condiciones habilitantes en las comunidades y abrir espacios de participación, sino que esta debe ser una participación cualificada y programática. Como mencionábamos, los procesos de gobernanza democrática no se limitan a abrir espacios de participación sino a garantizar la incidencia efectiva de los sectores vulnerables. Esto requiere una institucionalidad sensible y abierta al diálogo, pero también una sociedad civil organizada, con demandas claras y con estrategias adecuadas de visibilización, posicionamiento y debate. Es necesario crear una base teórica, generar capacidades para la planificación estratégica, elaborar planes de incidencia y apoyar financieramente su puesta en marcha desde las organizaciones de base. esto permitirá mejorar la capacidad de incidencia en los planes de desarrollo municipal, los РОт, los planes de sustitución de ilícitos, los PDET, etc. Estos modelos de fortalecimiento multinivel son condición necesaria para garantizar la legitimidad, representatividad y efectividad de los instrumentos de planificación del posconflicto.
4. Para promover la gobernanza democrática desde el ámbito local deben desarrollarse equilibrios entre la perspectiva institucional y las agendas ciudadanas, por lo que es muy importante la formación en liderazgo y el apoyo a los movimientos sociales comunitarios, muchas veces sustituidos o invisibilizados por las agendas sectoriales de los programas de desarrollo.

La cooperación europea, desde la implementación de los Laboratorios de Paz, ha entendido que es necesario apoyar las campañas ciudadanas como plataforma legitima y efectiva para la canalización de demandas y la incidencia política. Una de las estrategias de mayor impacto ha sido la puesta en marcha de escuelas teórico-prácticas de capacitación, como las escuelas de formación ciudadana, cuyo objetivo ha sido brindar herramientas a las organizaciones locales (formales o informales) para definir agendas estratégicas y planes de acción, y apoyar con recursos técnicos y financieros su materialización práctica. Para ello se han planteado dos modalidades, la inversión directa y el apoyo financiero a terceros. Muchas veces las líneas de financiación y los programas de desarrollo centran su atención en lo productivo y restringen el desarrollo social exclusivamente a la capacitación o a la participación ocasional. Los movimientos sociales de los entornos rurales y de las regiones posconflicto enfrentan grandes dificultades para posicionar sus agendas y canalizar sus demandas por medio de la institucionalidad, ya que la falta de recursos y las economías de subsistencia en las que están insertos les impide realizar estrategias de impacto para 
la visibilización y el posicionamiento de sus necesidades. El acompañamiento técnico es fundamental, y su pertinencia y efectividad se recoge en la experiencia del Canal del Dique, que concluye que:

La implementación de las Escuelas de Formación Ciudadana, a través de veinticinco réplicas de los talleres en los municipios, se convierte en uno de los grandes aciertos de la asistencia técnica para la población asociada. En ellas se abordaron diferentes temáticas como ciudadanía, derechos y deberes, convivencia y gestión de conflictos, participación ciudadana y mecanismos de exigibilidad del derecho, que se materializaron en la organización de diecisiete campańas de sensibilización con la participación de 1029 personas (Movimiento por la Paz, el Desarme y la Libertad, 2017).

Este componente de trabajo debe ser prioritario en el marco del posconflicto. Son los pobladores locales los que conocen las problemáticas que más les afectan por lo que, si se quiere construir paz desde la base, se deben destinar recursos técnicos y financieros para fortalecer modelos organizativos que articulen las demandas ciudadanas y las trasladen a las instancias de planificación y decisión. La presencia de los actores armados, la falta de institucionalidad y la ruptura del capital social en las regiones más afectadas por la guerra han impedido la organización comunitaria y el desarrollo de campańas ciudadanas, lo cual es una tarea pendiente y de vital importancia para el establecimiento de un nuevo diálogo social, la reducción de la desigualdad y las conflictividades, y la generación de condiciones para la convivencia pacífica.

\section{El fortalecimiento del capital social y el acercamiento entre comunidad e institu- cionalidad deben ser ejes transversales de trabajo con las comunidades más afectadas por el conflicto armado.}

El capital social no se reduce a la promoción de la asociatividad, sino que debe centrarse en la recuperación de confianza al interior de las comunidades, la búsqueda de nuevos diálogos sociales y la promoción de liderazgos comunitarios legítimos y representativos. Por ello, es necesario trabajar en nuevos modelos de relacionamiento a diferentes niveles. De la experiencia de Nuevos Territorios de Paz podemos destacar tres metodologías de trabajo. En primer lugar, los Grupos Autogestionados de Ahorro y Crédito, los cuales se convierten en un espacio en el cual los miembros no solo acuden a ahorrar o acceder a financiación, sino que las reuniones se convierten en espacios para compartir los problemas, buscar soluciones conjuntas y establecer instrumentos de ayuda mutua. Esta dinámica contribuye al fortalecimiento del tejido social dentro de la organización y, en muchos casos, a la dinamización de las actividades productivas (Unión Europea, Prosperidad Social y Red Adelco, 2015). En segundo lugar, el fortalecimiento de las juntas de acción comunal como plataformas efectivas para la canalización y representación de las demandas comunitarias. En tercer lugar, la apertura de espacios de diálogo, con participación protagónica de las comunidades, sobre las conflictividades que se presentan en el territorio. En el marco de NTP se combinaron ejercicios de formación en temáticas de derechos humanos y paz (con más de 2.000 personas) y la organi- 
zación de más de 50 espacios de diálogo en el territorio, que permitieron abordar de manera directa algunas de las problemáticas priorizadas por las comunidades, como los modelos económicos locales, el acceso y uso de la tierra o los conflictos políticos existentes. La participación conjunta de comunidades, representantes políticos y actores económicos no contribuye de manera directa al establecimiento de consensos, pero sí a la promoción de nuevos modelos de diálogo y convivencia, fundamentales para la consolidación de una paz duradera. Esto guarda estrecha relación con una de las conclusiones principales que recoge una de las investigaciones sobre construcción de paz desarrolladas en las cuatro regiones del país y que afirma que en términos generales, los resultados del diagnóstico territorial hacen evidente que uno de los retos más grandes que se presenta en el territorio es la recuperación de la confianza en el otro, pero sobre todo de la confianza institucional. Si bien hay instituciones mejor calificadas que representan no solo la confianza sino la generación de emociones positivas como el ejército, el reto aquí está en poder restablecer esa conexión entre ciudadanía y Estado, deteriorada por la violencia en el país. Como se pudo observar en los modelos, los individuos que participan comunitariamente y confían en el Gobierno tienen mayor confianza en el cumplimiento de los acuerdos que las personas que no lo hacen, por lo cual la confianza adquiere un rol fundamental en las acciones que se determinen por parte del Gobierno nacional. Esta confianza, como lo manifestaron los participantes, se construye con acciones y cumpliendo y trabajando conjuntamente (Corpovisionarios, 2017). En ese sentido, uno de los elementos clave de la intervención de NTP ha sido trabajar en un acercamiento entre sociedad civil e institucionalidad, sobre todo en aquellas regiones donde las relaciones antagónicas con el Estado han generado gran escepticismo ante el rol y la legitimidad de lo público. Quizás el elemento de mayor impacto en NTP ha sido que, a pesar de ser recursos de cooperación internacional, estos han sido canalizados y operados en muchos casos a través de una entidad del Gobierno nacional, el Departamento para la Prosperidad Social. Este modelo de intervención, tal como lo recoge la academia en su investigación "Comunidades constructoras de paz", fortaleció el ejercicio activo de la ciudadanía ya que los proyectos auspiciados con el apoyo de la cooperación de la Unión Europea, canalizados por Prosperidad Social, fortalecieron en las comunidades beneficiarias los saberes y las prácticas sociales que son la base de los incrementos de la productividad $y$, sobre todo, la base de la convivencia pacífica. En efecto, esos proyectos, al mismo tiempo que promueven iniciativas que impactan de manera positiva en el desarrollo de la región, fortalecen y reproducen los consensos sociales que suelen denominarse tejido social o capital humano, y que en los términos de los líderes de las comunidades suelen denominarse ejercicio activo de la ciudadanía (Universidad de Externado de Colombia, Unión Europea y Prosperidad Social, 2017).

\section{APUESTA DE SOSTENIBILIDAD}

El programa Nuevos Territorios de Paz, que permitió generar condiciones de paz y reducción de conflictividades en medio del 
conflicto armado, concluye durante la implementación de los acuerdos de paz y evidencia la necesidad de capitalizar y difundir sus aprendizajes como la principal estrategia de sostenibilidad. Existen planteamientos muy similares entre el modelo de intervención de NTP y los modelos de planificación e inversión planteados para el posconflicto, principalmente en lo que se refiere a la reforma rural integral, por lo que el programa supone un referente metodológico y operativo de gran relevancia. El presente artículo trató de visibilizar algunos de los enfoques y aprendizajes conceptuales acumulados, pero deberá ser materializado de manera más amplia en espacios técnicos de diálogo interinstitucional, iniciativas de transferencia metodológica e iniciativas de diseńo y formulación de nuevos proyectos y programas. Entre los relevos naturales del programa estará la nueva institucionalidad derivada de los acuerdos y competente en su implementación, las entidades territoriales que liderarán el proceso de posconflicto desde el ámbito local, y los actores de desarrollo, organizacionales y sociedad civil, que asumirán un papel protagónico en los procesos de planeación, seguimiento y gobernanza. Uno de los grandes retos que se identifican en los próximos meses será como operativizar las políticas públicas con enfoque territorial y sus modelos de intervención ya que, a pesar de que los PDET servirán como eje articulador de las inversiones públicas e internacionales, estos enfrentarán grandes retos para la movilización de los sectores vulnerables, la búsqueda de amplios consensos, y la integración de las agendas sectoriales y comunitarias en acuerdos territoriales de mayor alcance y cobertura. Podemos distinguir tres grandes dimensiones de vital importancia para la consolidación de los acuerdos de paz, y en las que los aprendizajes recogidos en el artículo serán de especial pertinencia. Por un lado, la movilización de los sectores vulnerables que, de manera organizada, cualificada y estratégica incidan en los instrumentos de planificación e inversión del posconflicto, bajo criterios de inclusión socioeconómica y reducción de las conflictividades. Por otro lado, el proceso de integración de dichos planes de acción en las políticas públicas territoriales y el proceso de articulación de los cooperantes en torno a esas agendas, con una mirada de complementariedad, pertinencia y eficacia. Por último, los modelos de gobernanza de esos planes territoriales que permitan avanzar hacia un nuevo relacionamiento entre institucionalidad y comunidades, sobre principios de dialogo social, gobernanza democrática y gestión transparente, y que garanticen el correcto cumplimiento de los acuerdos y el impulso de los consensos territoriales como estrategias de desarrollo y reconciliación en el largo plazo. De la mano de los actores regionales, y en estrecha colaboración con el Gobierno nacional, la Unión Europea ha puesto en marcha el Fondo Fiduciario para la Paz, que asume e integra los aprendizajes acumulados desde los Laboratorios de Paz, y que servirá de vehículo dinamizador de las estrategias de construcción de pazy reducción de las desigualdades que los propios actores locales identifiquen como prioritarias y estratégicas, principalmente en lo que compete a los puntos 1 y 3 de los acuerdos de paz. 


\section{REFERENCIAS}

Castañeda, D. (2017) La Unión Europea y la construcción de paz en Colombia. Como la cooperación internacional puede apoyar la transición del conflicto armado a la paz. Bogotá: Universidad de los Andes.

Corpovisionarios, Unión Europea y Prosperidad Social (2017). Tejiendo acciones de paz. Informe final. Bogotá.

Departamento Nacional de Planeación (2007). Pobreza rural: evaluación y diagnóstico de las políticas nacionales. Bogotá: DNP.

Departamento Nacional de Planeación (2013). Bases para la formulación de la politica nacional de desarrollo local. Bogotá: DNP.

Departamento Nacional de Planeación (2014). Misión para la transformación del campo. Marco conceptual de la misión. Bogotá: DNP.

Departamento Nacional de Planeación, Alta Consejería para el Posconflicto y Alto Comisionado para la Paz (2016). Los planes de desarrollo territorial como un instrumento de construcción de paz. Bogotá.

Fundación Universitaria Católica del Norte (2017). Estrategias de desarrollo rural integral. El caso del proyecto Promodique. Cartagena.

Gobierno de Colombia (2016). Acuerdo final para la terminación del conflicto y la construcción de una paz estable y duradera. Colombia.

González, J. (2016). Empresa privada: principal socio en el posconflicto y la construcción de paz. Revista Panorama, 10 (18), 84-92.

Guzmán, L. P. (20 de enero de 2017). El catastro multipropósito: retos y oportunidades para el desarrollo rural. La Silla Vacía. Recuperado de http://lasillavacia.com.

Hilfswerk Austria, Unión Europea y Prosperidad Social (2017). Compartir Saberes. Una estrategia inter- cultural de gestión del conocimiento e innovación comunitaria. Guaviare.

Hilfswerk Austria, Unión Europea y Prosperidad Social (2017). Innovación comunitaria intercultural. Encuentro de comunidades nativas y campesinas del Guaviare. Guaviare.

Maldonado, D. (2016). La participación ciudadana en la construcción de la paz territorial en Colombia. Bogotá: Redprodepaz, Cinep, Unión Europea y Prosperidad Social.

Ministerio de Agricultura y Desarrollo Rural (2017). Decreto 893 de 2017. Bogotá.

Perry, S. (2010). La pobreza rural en Colombia. Bogotá: RIMISP.

Perry, S. (2014). Seminario "Desarrollo Rural con Enfoque Territorialy su Institucionalidad". Bogotá: RIMISP.

Pitt, D. (1976). Development from Below: Anthropologists and Development Situations. Chicago: Walter De Gruyter Inc.

Presidencia de la República (2017). Acuerdo Final para la terminación del conflicto y la construcción de una paz estable y duradera. Bogotá.

Red Nacional de Agencias de Desarrollo Local, Unión Europea y Prosperidad Social (2015). Construcción de capital social con enfoque territorial. Bogotá.

Unión Europea, Prosperidad Social y Red Adelco (2016). Desarrollo Económico Local y Paz Territorial. Aprendizajes y reflexiones en contextos de posconflicto. Bogotá.

Universidad del Externado, Unión Europea y Prosperidad Social (2017). Comunidades Constructoras de Paz. Caquetá: sembramos convivencia. Bogotá.

Vigil, J. y Fernández, V. (2012). Gobernanza y regiones en perspectiva crítica: un abordaje para la construcción de políticas públicas. Revista del CLAD Reforma y Democracia, 53, 1-2. 